\title{
Comparison of CSWT and VSWT Aerodynamic Characteristics
}

\author{
Qiang Lu \\ Wind power R \& D department \\ NARI Technology Development Co., Ltd. \\ Nanjing, China \\ luqiang@sgepri.sgcc.com.cn
}

\author{
Zhen Luo \\ Wind power R \& D department \\ NARI Technology Development Co., Ltd. \\ Nanjing, China \\ luozhen@sgepri.sgcc.com.cn
}

\begin{abstract}
In this paper, we compare the aerodynamic characteristics of CSWT and VSWT. As an example, two 1MW wind turbines are selected and calculated based on the blade element momentum method and the aerodynamic characteristic of wind turbines by organizing the MATLAB simulation program is obtained. The results show that VSWT has better the power capture ability than CSWT before the rated wind speed. Toward the VSWT, it should be used in relatively large change of wind speed. Toward the CSWT, it has the advantages of simple structure, reliable property and low costs.
\end{abstract}

Index Terms-CSWT, VSWT, Blade Element Momentum Method, Aerodynamic Characteristic.

\section{NOMENCLATURE}

The air density.

The cross sectional area at the rotor disc.

The area in the far upstream.

The area in the far downstream.

The flow velocity.

The velocity at the rotor disc.

The velocity in the far downstream.

The relative wind velocity.

Axial induction factor

Tangential induced factor.

Static pressure.

Power coefficient.

Thrust coefficient.

Rotor rotational speed.

Radial coordinate at rotor plane.

Radius of wind turbine rotor.

Tip-speed ratio.

Produced power of wind turbine rotor.

The aerodynamic torque.

The thrust force.

The inflow angle.

The pitch angle.

The number of blades.

\section{INTRODUCTION}

Electricity generation using wind energy has been well recognized as environmentally friendly, economically competitive, and socially beneficial for many applications. The equipment of wind power in world is developing faster than expected. Nowadays, there are various sorts of wind turbine applied to generate electricity power. The major popular types are CSWT and VSWT.

The main difference between CSWT and VSWT is generator. In this paper, a squirrel-cage inductor generator is used in CSWT. And the CSWT can control the pitch to keep rotor in the synchronous speed in the high wind speed. On the other hand, VSWT use a doubly-fed induction generator [1]. It can generate electrical power within a wider range of speeds. Therefore, according the difference of the generator, we compare the aerodynamic characteristics of WT for designing the advanced control technology.

In the design of wind turbine, the main purpose is to improve the capacity of wind energy capture. To improve the power capture ability, the aerodynamic characteristics of WT are continuously improved. The Blade Element Momentum Method is a more precise engineering calculation method, and it is widely used in the wind energy industry. Usually, we used the BEM method to estimate the aerodynamic characteristics of the rotor and the output power from WT [2], [3]. In this paper, the MATLAB simulation program has been validated and developed by the comparison with the results of CSWT and VSWT. Based on the BEM method, the performances of CSWT and VSWT are compared. The results show that there 
are different the electrical power and the load in the different wind speed region.

Generally, the general WT operate in two main regions: the first one is the half load region, which the wind speed changes from the cut-in speed to the rated speed; the second one is the full load region, which the wind speed changes from the rated speed to the cut-out speed [4]. In the half load region, the main purpose is to improve ability to capture the power. In the full load region, the main purpose of the power control is as close as possible to the rated power [5]. In this study, we compare the aerodynamic performance of WT from these two main regions. Steady-state calculation is used to generate a power curve. It generates the wind turbine power curve as a function of wind speed, assuming uniform steady winds. The generator speed and electrical power, aerodynamic torque and thrust force are calculated.

\section{THE Blade ELEMENT MOMENTUM METHOD}

\section{A. Momentum theory}

Wind turbine to absorb the kinetic energy from the air that pass through the rotor disc. Wind speed will be decreased due to the kinetic energy transfer. The air was separated, and the cross section of the circular stream tube was obtained [6] (see Fig. 1).

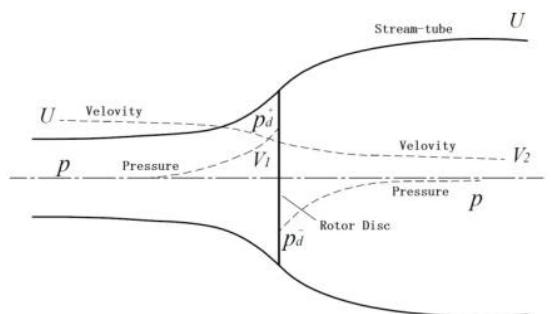

Fig. 1. An Energy Extracting Rotor Disc and Stream-tube.

The presence of the rotor disc led to the approaching air in the upstream gradually to slow down. Because the air mass flow rate along the stream-tube is equal, the reduction of wind speed leads to the expansion of the stream-tube. Because no work has yet been done on the air, the static pressure of air will be going on. The static pressure drop is generated by the air passing through the rotor disc. This results in reduced speed and static pressure. This flow region is called a wake. Eventually, to maintain balance, the pressure in far downstream must achieve to consistent with the atmospheric level.

The mass flow rate must be the same everywhere along the stream-tube and so

$$
\rho A_{1} U=\rho A V_{1}=\rho A_{2} V_{2}
$$

It is generally consider that the rotor disc induces a freestream velocity variation. It is defined that the axial induction factor is $a$. At the disc, therefore, the velocity at the rotor disc is

$$
V_{1}=(1-a) U
$$

The force caused by this momentum change comes entirely from the pressure differential across the actuator disc. Therefore,

$$
\begin{aligned}
& T=\left(p_{d}^{+}-p_{d}^{-}\right) A=\rho A V_{1}\left(U-V_{2}\right) \\
& =\rho A U(1-a)\left(U-V_{2}\right)
\end{aligned}
$$

Assuming the air to be incompressible, Bernoulli's equation is applied to a unit volume of air in the stream-tube. Thus,

$$
p_{d}^{+}-p_{d}^{-}=\frac{1}{2} \rho\left(U^{2}-V_{2}^{2}\right)
$$

By combining Equation (3) and (4), we obtain:

$$
V_{2}=(1-2 a) U
$$

Equation (2) and (5) show that the loss of the axial velocity in the stream-tube half in the upstream of the rotor disc and half in the downstream. (5)

The thrust force on the air becomes, from Equation (3) and

$$
T=2 \rho A U^{2} a(1-a)
$$

This force doing work on the rotor disc, and the output power is given by

$$
P=T V_{1}=2 \rho A U^{3} a(1-a)^{2}
$$

A power coefficient is then defined as

$$
C_{p}=\frac{P}{\frac{1}{2} \rho A U^{3}}=4 a(1-a)^{2}
$$

When $a=1 / 3$, the $C_{p}$ is at its maximum, and $C_{P \max }=0.593$. The maximum achievable value of the power coefficient is known as the Betz limit. Similarly, the thrust coefficient,

$$
C_{T}=\frac{T}{\frac{1}{2} \rho A U^{2}}=4 a(1-a)
$$

The energy obtained in the plane of rotor which is translated into rotor rotational speed and aerodynamic torque. When the airflow passing through the rotor disc. The torques impacting on the rotor and airflow are equal and opposite. Reverse torque makes the airflow obtained the velocity component in the tangential direction. The airflow has no 
rotational motion at all before it enters the rotor disc and does have rotation after it exits the rotor disc. The tangential velocity changes in the rotor disc. Therefore, it is expressed in terms of a tangential induced factor $a^{\prime}$. Upstream of the disc the tangential velocity is zero. Immediately downstream of the disc the tangential velocity is $2 \Omega \mathrm{ra}^{\prime}$. At the middle of the disc thickness, a radial distance from the axis of rotation, the induced tangential velocity is $\Omega r a^{\prime}$.

At the edge of the disc $r=R$, the tip speed ratio is

$$
\lambda=\frac{\Omega R}{U}
$$

The aerodynamic torque on an annulus of radius and radial width is

$$
\begin{aligned}
& Q=\int_{0}^{R} 4 \pi \rho U(1-a) a^{\prime} \Omega r d r \\
& =4 \pi \rho U(1-a) a^{\prime} \Omega R^{4}
\end{aligned}
$$

\section{B. Blade element theory}

Wind turbine blade is divided into a finite number of blade elements along the radial superposition in Blade element theory. Assuming air is flowing without mutual interference between each blade element, it is regarded as a 2D dimensional airfoil. Then the aerodynamic on the blade is obtained by computing the integral for each element aerodynamic [7].

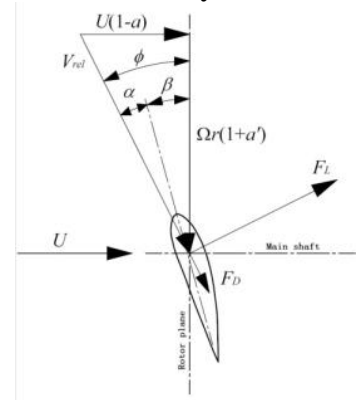

Fig. 2. Flow diagram of a blade element

As shown in Fig. 2, The relative wind is the vector sum of the wind velocity at the rotor, $U(1-a)$, and the wind velocity due to rotation of the blade. This rotational component is the induced angular velocity at the blades from conservation of angular momentum, $\Omega r\left(1+a^{\prime}\right)$. Finally, $V_{r e l}$ is the relative wind velocity. And the inflow angle is

$$
\phi=\arctan \left[\frac{U(1-a)}{\Omega r\left(1+a^{\prime}\right)}\right]
$$

Set the chord length of blade element is $c$. The lift and drag of the blade element separately are

$$
\begin{gathered}
F_{L}=\frac{1}{2} \rho V_{r e l}^{2} C_{L} c d r \\
F_{D}=\frac{1}{2} \rho V_{r e l}^{2} C_{D} c d r
\end{gathered}
$$

Then, the thrust force on the radius is

$$
\begin{aligned}
& d T=B\left(F_{L} \cos \phi+F_{D} \sin \phi\right) \\
& =\frac{1}{2} \rho V_{r e l}^{2}\left(C_{L} \cos \phi+C_{D} \sin \phi\right) B c d r
\end{aligned}
$$

The aerodynamic torque is

$$
\begin{aligned}
& d Q=B\left(F_{L} \sin \phi-F_{D} \cos \phi\right) r \\
& =\frac{1}{2} \rho V_{r e l}^{2} r\left(C_{L} \sin \phi-C_{D} \cos \phi\right) B c d r
\end{aligned}
$$

\section{The blade element-momentum (BEM) theory}

Here, we calculate the induction factors by using the iteration method. Now it is the batter method in engineering application [8] (see Fig. 3). In the calculation of induction factors, $a$ and $a^{\prime}$, we should use the torque equations from momentum and blade element theory. By combining Equation (6) and (15), (11) and (16), we obtain

$a=\frac{B c\left(C_{L} \cos \phi+C_{D} \sin \phi\right)}{8 \pi r \sin ^{2} \phi+B c\left(C_{L} \cos \phi+C_{D} \sin \phi\right)}$

$a^{\prime}=\frac{B c\left(C_{L} \sin \phi-C_{D} \cos \phi\right)}{8 \pi r \sin \phi \cos \phi-B c\left(C_{L} \sin \phi-C_{D} \cos \phi\right)}$

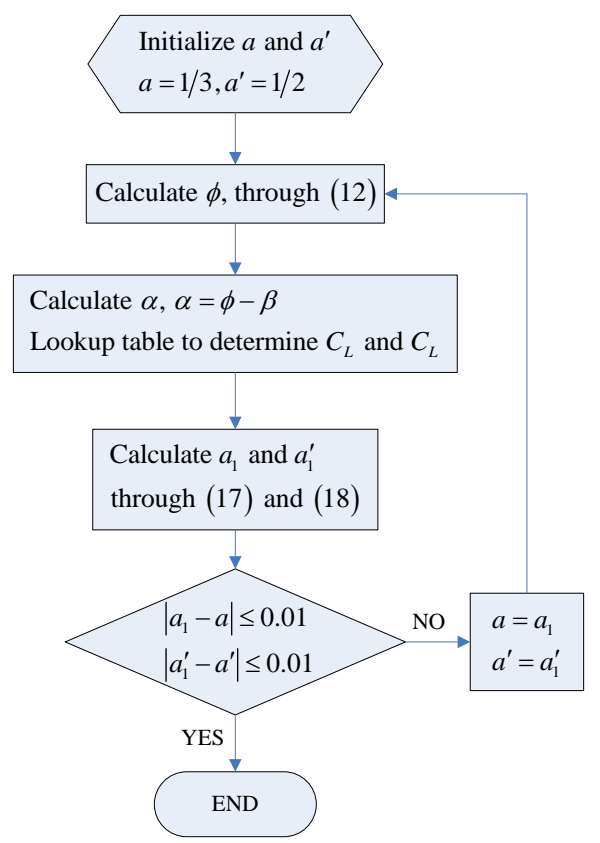

Fig. 3. The Calculating Flow Chart 


\section{EXAMPLE AND RESULTS}

\section{A. Example model}

In order to demonstrate the formulated calculation model in the MATLAB simulation program, we take the CSWT and VSWT as example. The rated power of this two WT is $1 \mathrm{MW}$, and the other detailed parameters are shown in Tab.1. And Fig.4 shows lift and drag coefficient, based on the experimental 2D section data for the NACA 63-215 airfoil, which was obtained at the National Advisory Committee for Aeronautics (NACA) with a Reynolds number of 2,000,000 [9].

TABLE I. THE PARAMETERS OF CSWT AND VSWT

\begin{tabular}{|l|l|l|}
\hline \multicolumn{1}{|c|}{ Parameters } & \multicolumn{1}{c|}{ CAWT } & \multicolumn{1}{c|}{ VSWT } \\
\hline Section airfoils & NACA 63-215 & NACA 63-215 \\
\hline Length of blade (m) & 31 & 31 \\
\hline Diameter of rotor (m) & 65 & 65 \\
\hline Rated generator speed (rpm) & 1500 & 1800 \\
\hline Rated power (kW) & 1000 & 1000 \\
\hline Cut-in wind speed (m/s) & 4.5 & 3.5 \\
\hline Cut-out wind speed (m/s) & 25 & 25 \\
\hline
\end{tabular}

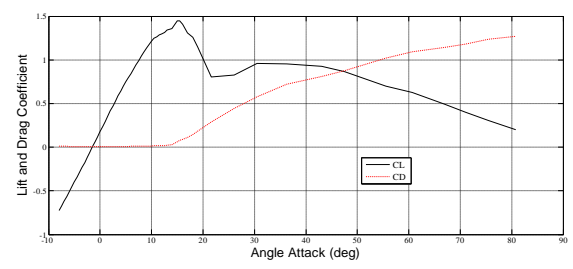

Fig. 4. The $C_{L}$ and $C_{D}$ of NACA 63-215 airfoil

\section{B. Results}

According to the different of the generator characteristics, the WT connects grid at the different speed, as shown in Fig.5. Because CSWT is synchronous generator, it keeps the generator speed around the synchronous speed. The results show that the VSWT connect grid at $1080 \mathrm{rpm}$, and it is running at rated speed above the rated wind speed. This is the essential difference between CSWT and VSWT.

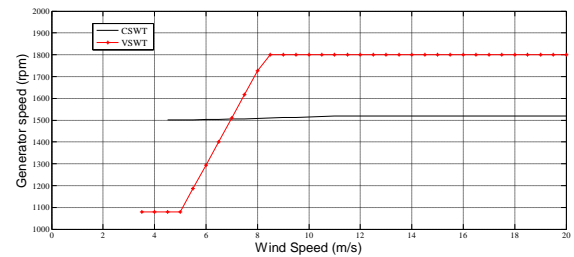

Fig. 5. The Generator Speed of CSWT and VSWT

Fig.6, Fig.7 and Fig.8 show the performances in terms of produced power of CSWT and VSWT. We can see that:

- The VSWT's power coefficient is greater than CSWT.

And it has an optimal $C_{p}$ value in the half load region. However, they are basically the same in the full load region.

- The tip speed ratio is similar to power coefficient. It has a same constant region in the half load region.
- Toward the electric power, the VSWT is better than the CSWT in half load region. But it rarely improves the power capture ability.

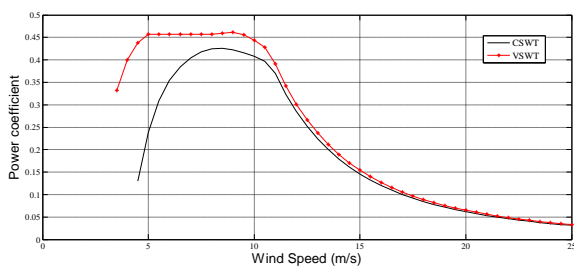

Fig. 6. The Power coefficient of CSWT and VSWT

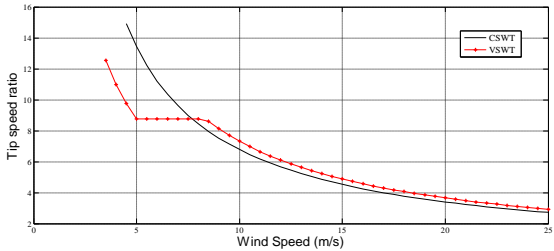

Fig. 7. The Tip Speed Ratio of CSWT and VSWT

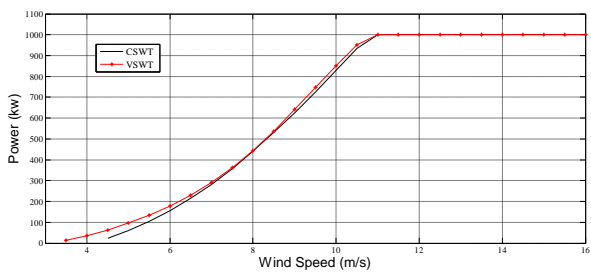

Fig. 8. The Electric Power of CSWT and VSWT

As seen from Fig.9, Fig.10 and Fig.11, we compare the loads acting on the WT rotor blade, and find that the VSWT's thrust coefficient $\mathrm{Ct}$ is greater than CSWT in the half load region. So it has to bear more thrust force. Moreover, the aerodynamic torque of VSWT is slightly less than CSWT in the full load region.

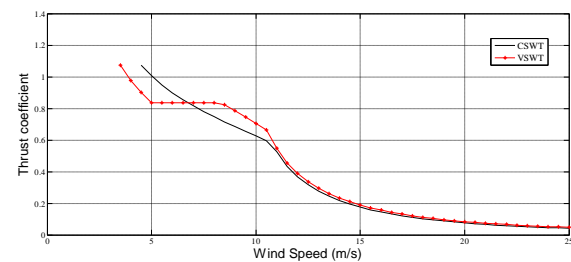

Fig. 9. The Thrust coefficient of CSWT and VSWT

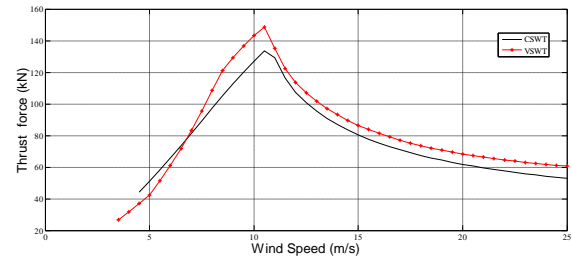

Fig. 10. The Thrust Force of CSWT and VSWT 


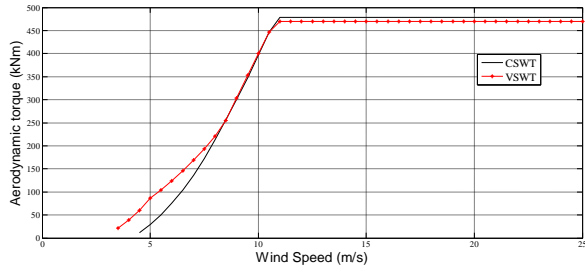

Fig. 11. The Aerodynamic Torque of CSWT and VSWT

\section{CONCLUSION}

In the present work, based on the BEM method, the MATLAB simulation program is obtained. By comparing the simulated results of the 1MW WT, the simulation model was formulated to compute the performance of the CSWT and VSWT. Due to the essence of the power generation method, they have the following different points:

- Through the above results analysis, we know that VSWT can capture more energy in the low wind speed. According the change of wind speed, VSWT can maintain the optimal tip speed ratio for capturing maximum wind energy in the half load region. However, in the high wind speed, the results show that the VSWT's power coefficient, tip speed ratio and electric power are the same as CSWT.

- $\quad$ To increase the power capture ability, the VSWT just withstand a greater thrust load. It will affect the lifetime of the WT.

- In other words, the CSWT has advantages of simple structure, low costs, reliable property and the long service life, etc.

Moreover, there are a lot of work needs to be done in the area of improving ability to capture the power. The aerodynamic characteristics of the WT are the most fundamental determinants of the ability to capture the power. Toward the different kinds of WT, the more important work in the future is analyzing the relationship between the aerodynamic characteristics of WT and improvement of produced power.

\section{REFERENCES}

[1] Olimpo Anaya-Lara, Nick Jenkins, Janaka Ekanayake, et al. Wind Energy Generation: Modelling and Control. West Sussex, England: John Wiley \& Sons Ltd, 2009.

[2] R. Lanzafame, "Fluid dynamics wind turbine design: Critical analysis, optimization and application of BEM theory," Renewable energy, vol. 32, 2007, pp. 2291-2305.

[3] J. Dai, Y. Hu, D. Liu, and X. Long, "Aerodynamic loads calculation and analysis for large scale wind turbine based on combining BEM modified theory with dynamic stall model," Renewable energy, vol. 36, 2011, pp.1095-1104.

[4] L. Zhang, H. Li, C. E, J. Li, and H. Xu, "Pitch control of large scale wind turbine based on fuzzy-PD method," A tutorial review, Proc. 3th International Conf. on Deregulation and Restructuring and Power Technologies, Nanjing, China, 2008, pp. 2447-2452.

[5] Weijun WANG, "Comparison of Six Horizontal Axis Wind Turbines," A tutorial review, Proc. 11th International Conf. on Environment and Electrical Engineering, Venice, Italy, 2012, pp. 419-424.

[6] Tony Burton, David Sharpe, Nick Jenkins, et al. Wind energy Handbook. West Sussex, England: John Wiley \& Sons Ltd, 2001.

[7] J.F. Manwell, J.G. McGowan, A.L. Rogers, Wind energy Explained. West Sussex, England: John Wiley \& Sons Ltd, 2002.

[8] M. L. Buhl, "A New Empirical Relationship between Thrust Coefficient and Induction Factor for the Turbulent Windmill State," Tech. Rep. NREL/TP-500-36834, National Renewable Energy Laboratory (NREL), 2005.

[9] Björk, A., "A Guide to Data Files From Wind Tunnel Test of A NACA 63-215 Airfoil at FFA," Report FFAP-V-018, 1996. 